\title{
Black-sun noise immune correlated double sampling scheme for CMOS image sensors
}

\author{
Je-Hoon Lee ${ }^{1}$ and Hyeon-June Kim ${ }^{1}$, a)
}

Abstract This paper presents a black-sun immune correlated double sampling (CDS) scheme for high-quality imaging. Based on an analysis of signal characteristics in strong light conditions, a clamping circuit-based signal difference generator is proposed to accurately present the bright light information. The proposed scheme eliminates the black-sun noise with simple circuitry to improve the A/D conversion efficiency. Moreover, it can be is reversible to the conventional algorithm so that it still preserves the structural advantages of the existing CMOS image sensor (CIS) structure. A prototype CIS with a column-parallel 11-bit single-slope (SS) analogto-digital converter (ADC) was fabricated in a $0.11-\mu \mathrm{m} 1 \mathrm{P} 4 \mathrm{M}$ CIS process with a $2.9-\mu \mathrm{m}$ pixel pitch

Keywords: CMOS imager sensor, black-sun noise, correlated double sampling, single-slope analog digital converter

Classification: Integrated circuits (memory, logic, analog, RF, sensor)

\section{Introduction}

Recently, CISs have received considerable attention for a variety of applications $[1,2,3,4,5,6]$. Owing to the increasing demand for high-quality images, various CIS structures with low-noise performance have been developed [7, 8, 9, 10]. In commercial CISs, a SS ADC structure with a 4-transistor active pixel sensor (4T-APS) is employed for its structural advantages [11, 12, 13] that it can adopt correlated double sampling (CDS) scheme during the pixel readout operation to eliminate fixed pattern noise (FPN) $[14,15,16]$. However, when the initial value is contaminated, it is difficult to obtain an accurate signal value because the voltage difference obtained is considered as the signal value. As reported in previous studies [17, 18, 19, 20], in strong light illumination conditions, the photo-charge overflows from a photodiode (PD) to a floating node (FD). This causes the initial value of the pixel to drop abnormally during the CDS operation resulting in output image errors (i.e., black-sun noise). To solve this problem, in [17] a new readout scheme was proposed to eliminate black-sun noise.

However, due to additional circuitries, the circuit area and design complexity also increased. In this paper, an efficient black-sun immune CDS scheme is proposed with a novel signal difference generator (SDG). The proposed scheme maintains the existing commercial structure and readout op-

\footnotetext{
${ }^{1}$ Department of Electrical Information Communication Engineering, Kangwon National University, 1 Joongang-ro, Samcheok, Gangwon, 245-711, Republic of Korea

a) hyeonjunekkim@kangwon.ac.kr
}

DOI: $10.1587 /$ elex.18.20210175

Received April 15, 2021

Accepted April 20, 2021

Publicized April 28, 2021

Copyedited May 25, 2021 eration form, thereby presenting several commercial advantages.

\section{Proposed black-sun immune CDS scheme}

Fig. 1 shows a simplified schematic of a conventional SS ADC with the proposed SDG. The SDG is connected to the pixel output $\left(\mathrm{V}_{\mathrm{PX}}\right)$ node. It consists of an analog switch (Mux.), two signal voltages $\left(\mathrm{V}_{\mathrm{SELB}}\right.$ and $\left.\mathrm{V}_{\mathrm{REF}}\right)$, and current bleeding circuits $\left(\mathrm{M}_{\mathrm{PC}}, \mathrm{M}_{\mathrm{SB}}\right.$, and $\left.\mathrm{M}_{\mathrm{EN}}\right)$. It is based on the $\mathrm{V}_{\mathrm{PX}}$ common generator [21], which is used only after completing the pixel readout operation. According to rolling shutter techniques [22], when the $\mathrm{V}_{\mathrm{PX}}$ node is changed from $\mathrm{V}_{\text {SIG(N-1) }}$ to $\mathrm{V}_{\mathrm{RST}(\mathrm{N})}\left(\mathrm{V}_{\mathrm{SEL}}\right.$ is off), $\mathrm{M}_{\mathrm{SB}}$ is switched by $\mathrm{V}_{\text {SELB }}$ and $V_{P X}$ remains constant at a certain voltage level, which reduces $\mathrm{V}_{\text {PX }}$ fluctuation. Here, $\mathrm{V}_{\text {RST }}$ and $\mathrm{V}_{\text {SIG }}$ represent a pixel reset voltage and pixel signal voltage, respectively.

In this study, the SDG is utilized to achieve an additional performance. When pixel readout operation starts $\left(\mathrm{V}_{\mathrm{SEL}}\right.$ is on), $\mathrm{M}_{\mathrm{SB}}$ is biased to a predetermined voltage $\left(\mathrm{V}_{\mathrm{REF}}\right)$. Subsequently, it observes whether $V_{P X}$ is lower than $V_{R E F}-$ $\mathrm{V}_{\mathrm{TH}}$ and prevent a drop in $\mathrm{V}_{\mathrm{PX}}$, as a the $\mathrm{V}_{\mathrm{PX}}$-clamping circuit. Here, $\mathrm{V}_{\mathrm{REF}}$ can be set at the $\mathrm{V}_{\mathrm{PX}}$-clamping level, $\mathrm{V}_{\mathrm{TH}}$ is the threshold voltage for $\mathrm{M}_{\mathrm{SB}}$.

The operational timing diagram and those waveforms are illustrated in Fig. 2 (a) and (b), respectively. For the pixels chosen by $\mathrm{V}_{\mathrm{SEL}}, \mathrm{V}_{\mathrm{PX}}$ becomes $\mathrm{V}_{\mathrm{RST}}$ and $\mathrm{V}_{\mathrm{SIG}}$ for the pixel control signals of $\mathrm{V}_{\mathrm{RX}}$ and $\mathrm{V}_{\mathrm{TX}}$, respectively. $\mathrm{V}_{\mathrm{PX}}$ and $\mathrm{V}_{\mathrm{RAMP}}$ pass through $\mathrm{AC}$-coupling capacitors $\left(\mathrm{C}_{\mathrm{SN}}\right.$ and $\mathrm{C}_{\mathrm{SP}}$ ) and into comparator input nodes ( $\mathrm{V}_{\mathrm{INN}}$ and $\left.\mathrm{V}_{\mathrm{VINP}}\right)$, respectively. To perform a dual CDS $[23,24]$, the analog CDS $[25,26]$ is performed when $\mathrm{V}_{\mathrm{AZ}}$ is on while the digital CDS $[27,28]$ is performed twice, for both $\mathrm{V}_{\mathrm{RST}}$ and $\mathrm{V}_{\text {SIG }}$ when $V_{A / D}$ is on. Note that the initial value $\left(V_{R S T}\right)$ should be maintained until it changes to the signal value $\left(\mathrm{V}_{\mathrm{SIG}}\right)$ to

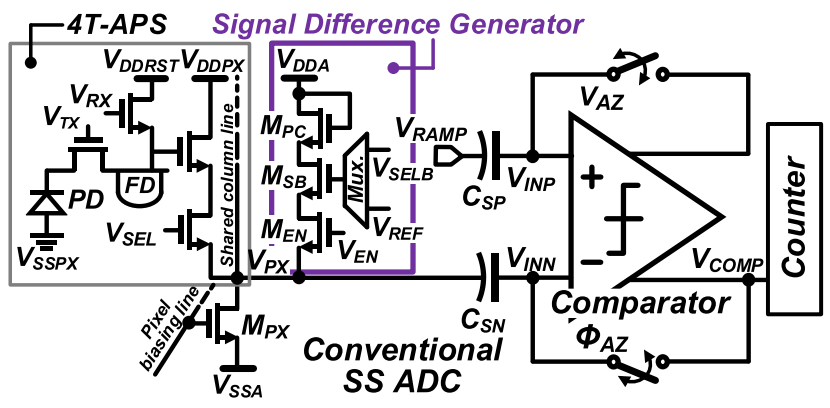

Fig. 1 Simplified schematic of the proposed column-parallel SS ADC. 


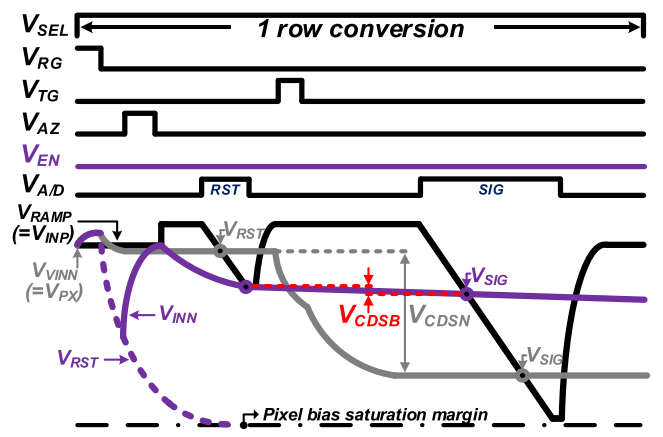

(a)

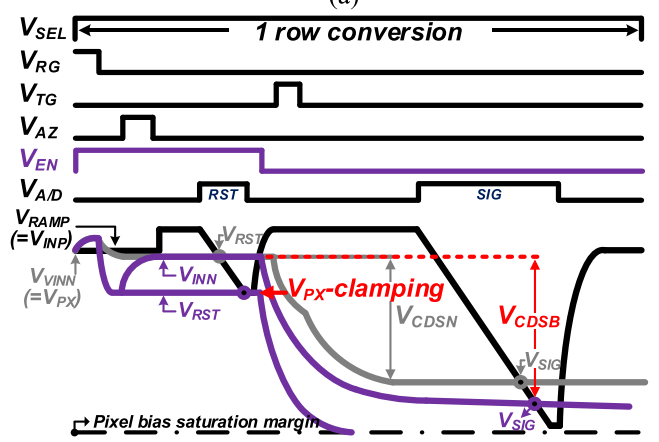

(b)

Fig. 2 Operational timing diagrams (a) without and (b) with the proposed CDS scheme in strong light illuminations

obtain an accurate difference $\left(\mathrm{V}_{\mathrm{CDS}}\right)$. However, in strong light illumination conditions (as plotted by the blue dashed line in Fig. 2 (a)), $\mathrm{V}_{\text {RST }}$ drops to a low voltage level and produces an incorrect CDS result $\left(\mathrm{V}_{\mathrm{CDSB}}<\mathrm{V}_{\mathrm{CDSN}}\right)$. Consequently, dark spots are formed within the bright region of the captured image; this is because $\mathrm{V}_{\mathrm{RST}}$ is not maintained at a constant level just before $\mathrm{V}_{\mathrm{TX}}$ is switched on. To solve this problem, the SDG is enabled ( $\mathrm{V}_{\mathrm{EN}}$ is on) right before $\mathrm{V}_{\mathrm{TX}}$ is switched on; furthermore, whether $\mathrm{V}_{\mathrm{RST}}$ is lower than $V_{R E F}-V_{T H}$, is observed. As shown in Fig. 2 (b), if $\mathrm{V}_{\mathrm{RST}}<\mathrm{V}_{\mathrm{REF}}-\mathrm{V}_{\mathrm{TH}}$, the SDG clamps $\mathrm{V}_{\mathrm{RST}}$ at approximately $\mathrm{V}_{\mathrm{REF}}-\mathrm{V}_{\mathrm{TH}}$. While the SDG is disabled ( $\Phi_{\mathrm{EN}}$ is off $)$, the maximum signal difference $\left(\mathrm{V}_{\mathrm{CDSB}} \geq \mathrm{V}_{\mathrm{CDSN}}\right)$ is generated which represents the bright light information. In this manner, the black-sun noise can be effectively eliminated during the dual CDS operation while maintaining the existing CIS structure and operational timing.

\section{Implementation and experimental results}

Fig. 3 shows the prototype chip and CIS test board. The prototype chip is fabricated using a $0.11-\mu \mathrm{m}$ CIS process with a dimension of $4.15 \times 2.55 \mathrm{~mm}^{2}$. It has a pixel array of $1024 \times 300$ with $2.9 \mu \mathrm{m}$-pitch 4T-APS. The prototype CIS chip demonstrates 210 frames per second (fps) with a power consumption of $30.5 \mathrm{~mW}$. The test environment for the prototype CIS is comprised a chip and field programmable gate array (FPGA) board. Here, an off-chip FPGA is used to generate multiple control signals for the prototype CIS. The captured image information (the output digital signals) is transferred to the computer using a USB interface and displayed through the Microsoft foundation class library (MFC)-based interface program. To evaluate

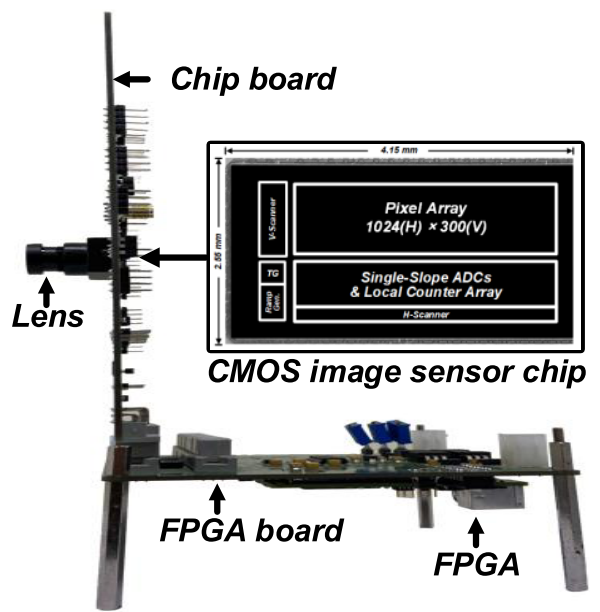

Fig. 3 Test environment with a fabricated chip and CIS test board.

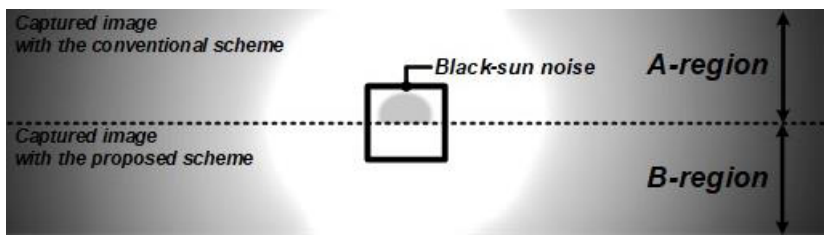

Fig. 4 Captured image from the prototype CIS.

Table I Comparison table.

\begin{tabular}{|c|c|c|c|c|c|}
\hline & $\underset{[30]}{\text { ISSCC'18 }}$ & $\begin{array}{c}\text { TCAS-I'20 } \\
{[31]}\end{array}$ & $\begin{array}{c}\text { JSSC'20 } \\
{[32]}\end{array}$ & $\begin{array}{c}\text { TED'21 } \\
{[17]}\end{array}$ & This work \\
\hline Process $(\mu \mathrm{m})$ & 0.09 & 0.11 & 0.11 & 0.11 & 0.11 \\
\hline Pixel resolution & $2560 \times 1536$ & $128 \times 128$ & $640 \times 640$ & $1024 \times 300$ & $1024 \times 300$ \\
\hline Frame rate (fps) & 60 & 120 & 44 & 280 & 210 \\
\hline ADC type & SS & SS & SS & SS & SS \\
\hline ADC resolution (bit) & 10 & 10 & 10 & 11 & 11 \\
\hline Random noise $\left(\mathrm{e}_{\mathrm{rms}}^{-}\right)$ & 1.8 & 5.01 & 6.1 & 2.18 & 2.03 \\
\hline Power consumption (mW) & 95 & 40 & 2.1 & 65.4 & 30.5 \\
\hline Black-sun elimination & $\mathbf{x}$ & $\mathbf{x}$ & $\mathbf{x}$ & 0 & 0 \\
\hline${ }^{1)} \mathrm{FoM}_{1}\left[\mathrm{e}^{-} \cdot \mathrm{pJ} / \mathrm{step}\right]$ & 0.71 & 52.33 & 0.69 & 0.81 & 0.47 \\
\hline${ }^{2)} \mathrm{FoM}_{2}\left[\mathrm{e}^{-/ \mathrm{MHz} / \mathrm{step}]}\right.$ & 19.07 & 167.6 & 211.5 & 12.7 & 15.7 \\
\hline
\end{tabular}

1) $\mathrm{FoM}_{1}=[$ Noise $] \times[$ Power consumption $] /[$ The number of pixels $] /[$ Frame rate $] / 2^{\wedge}$
2) $\mathrm{FoM}_{2}=[\mathrm{Noise}] /$ The number of vertical pixels $] /\left[\mathrm{Frame}\right.$ rate] $/ 2^{\wedge}[\mathrm{ADC}$ resolution $]$

the prototype chip in various test environments, $V_{\mathrm{REF}}$ is provided through an external DAC mounted on the CIS test board.

Fig. 4 shows the captured image while a bright light source [29] over approximately 100,000 lux is focused on the prototype CIS. Note that the measurements were taken at room temperature using an infrared-cut filter. To verify the effects of the proposed black-sun immune CDS scheme while extracting captured images, the proposed scheme was applied to only half of the rows (represented by B-region) and the other half was left unchanged (represented by Aregion). In A-region, because dark spots are more visible with the bright surroundings, the image quality seems to be worse. In contrast, the dark spots in B-region are eliminated while maintaining the original image quality.

Table I compares the performance of the current study with recently published works $[17,30,31,32]$. For a fair performance comparison, the figure of merits (FoMs) are calculated according to [33, 34]. Compared to [17], it requires an additional area of approximately $2.9 \times 110 \mu \mathrm{m}^{2}$, however, the proposed CDS scheme can be applied to the 
existing CIS structure and control-timing which results in a better area efficiency. Moreover, the prototype CIS with proposed CDS scheme demonstrated elimination of the blacksun noise while maintaining structural advantages over a commercial CIS.

\section{Conclusion}

In this study, a black-sun immune CDS readout scheme is proposed for high-quality imaging in strong light conditions. The proposed CDS scheme eliminated the black-sun noise without additional complex circuitry and operational timing modifications which degrades the A/D conversion efficiency. Moreover, the proposed scheme could be easily reversed to use the conventional SS A/D algorithm, thus, it can be effectively utilized in various commercial CIS applications as one of functions to improve image quality.

\section{Acknowledgments}

The chip fabrication and EDA tool were supported by the IC Design Education Center (IDEC), Korea. This study has been worked with the support of a research grant of Kangwon National University in 2016, and in part by the National Research Foundation of Korea (NRF) Grant funded by the Korean Government (MSIT) under Grant 2020R1I1A3074020.

\section{References}

[1] J. Ohta: Smart CMOS Image Sensors and Applications (CRC Press, Boca Raton, 2010) 2nd ed.

[2] A. Khosla and D.S. Kim: Optics Imaging Devices (CRC Press, Boca Raton, 2015).

[3] M. Amjad, et al:: "Wireless multimedia cognitive radio networks: a comprehensive survey," IEEE Commun. Surveys Tuts. 20 (2018) 1056 (DOI: 10.1109/COMST.2018.2794358).

[4] D. Bol, et al.: "Green SoCs for a sustainable Internet-of-Things," IEEE FTFC (2013) 1 (DOI: 10.1109/FTFC.2013.6577767)

[5] H. Kim: "CMOS image sensor for wide dynamic range feature extraction in machine vision," IET Electron. Lett. (2020) (DOI: 10.1049/ ell2.12087).

[6] T. Hsu, et al:: "5.9 A $0.8 \mathrm{~V}$ multimode vision sensor for motion and saliency detection with ping-pong PWM pixel," IEEE ISSCC Dig. Tech. Papers (2020) 110 (DOI: 10.1109/ISSCC19947.2020. 9062926).

[7] J. Cheon and G. Han: "Noise analysis and simulation method for a single-slope ADC with CDS in a CMOS image sensor," IEEE Trans. Circuits Syst. I, Reg. Papers 55 (2008) 2980 (DOI: 10.1109/ TCSI.2008.923434)

[8] J. Park, et al.: "A high-speed low-noise CMOS image sensor with 13b column-parallel single-ended cyclic ADCs," IEEE Trans. Electron Devices 56 (2009) 2414 (DOI: 10.1109/TED.2009.2030635).

[9] M. Kobayashi, et al.: "4.5 A 1.8erms- temporal noise over $110 \mathrm{~dB}$ dynamic range $3.4 \mu \mathrm{m}$ pixel pitch global shutter CMOS image sensor with dual-gain amplifiers, SS-ADC and multiple-accumulation shutter," ISSCC Dig. Tech. Papers (2017) 74 (DOI: 10.1109/ISSCC.2017. 7870267).

[10] "Samsung to Challenge Sony. CMOS Image Sensor New Technology 'Competition,", http://www . epnc.co.kr/news/articleView. html?idxno=75952.

[11] E.R. Fossum and D.B. Hondongwa: "A review of the pinned photodiode for CCD and CMOS image sensors," IEEE J. Electron Devices Soc. 2 (2014) 33 (DOI: 10.1109/JEDS.2014.2306412).

[12] A.J.P. Theuwissen: "Better pictures through physics," IEEE SolidState Circuits Mag. 2 (2010) 22 (DOI: 10.1109/MSSC.2010.936662).

[13] I. Inoue, et al.: "Low-leakage-current and low-operating-voltage buried photodiode for a CMOS imager," IEEE Trans. Electron Devices 50 (2003) 43 (DOI: 10.1109/TED.2002.807525).

[14] K. Yonemoto, et al.: "A CMOS image sensor with a simple FPNreduction technology and a hole accumulated diode," ISSCC Dig. Tech. Papers (2000) 102 (DOI: 10.1109/ISSCC.2000.839709).

[15] S. Yoshihara, et al.: "A 1/1.8-inch 6.4 Mpixel 60 frames/s CMOS image sensor with seamless mode change,” IEEE J. Solid-State Circuits 41 (2006) 2998 (DOI: 10.1109/JSSC.2006.884868).

[16] P. Holloway: "A trimless $16 \mathrm{~b}$ digital potentiometer," ISSCC Dig. Tech. Papers (1984) 66 (DOI: 10.1109/ISSCC.1984.1156642).

[17] H.-J. Kim: "A sun-tracking CMOS image sensor with black-sun readout scheme," IEEE Trans. Electron Devices 68 (2021) 1115 (DOI: 10.1109/TED.2021.3052450).

[18] R. Saleem, et al.: "A cost-effective micro sun sensor based on black sun effect,” 2017 IEEE SENSORS (2017) 1 (DOI: 10.1109/ICSENS. 2017.8234366)

[19] Product datasheet KAC-06040, https://www.onsemi.com/pub/ Collateral/KAC-06040-D.PDF.

[20] S. Rashid and L. Sukhan: "Accurate and cost-effective micro sun sensor based on CMOS black sun effect," Sensors 19 (2019) 739 (DOI: 10.3390/s19030739).

[21] H.-J. Kim: "11-bit column-parallel single-slope ADC with first-step half-reference ramping scheme for high-speed CMOS image sensors," IEEE J. Solid-State Circuits 55 (2021) 1 (DOI: 10.1109/jssc. 2021.3059909).

[22] C. Liang, et al:: "Analysis and compensation of rolling shutter effect," IEEE Trans. Image Process. 17 (2008) 1323 (DOI: 10.1109/TIP. 2008.925384).

[23] Y. Nitta, et al:: "High-speed digital double sampling with analog CDS on column parallel ADC architecture for low-noise active pixel sensor," ISSCC Dig. Tech. Papers (2006) 2024 (DOI: 10.1109/ ISSCC.2006.1696261).

[24] T. Arai, et al.: "A 1.1 $\mu \mathrm{m}$ 33Mpixel 240fps 3D-stacked CMOS image sensor with 3-stage cyclic-based analog-to-digital converters," ISSCC Dig. Tech. Papers (2016) 126 (DOI: 10.1109/ISSCC.2016.7 417939).

[25] H. Kim, et al.: "A delta-readout scheme for low-power CMOS image sensors with multi-column-parallel SAR ADCs," IEEE J. Solid-State Circuits 51 (2016) 2262 (DOI: 10.1109/JSSC.2016.2581 819).

[26] H. Kim, et al.: "A dual-imaging speed-enhanced CMOS image sensor for real-time edge image extraction,” IEEE J. Solid-State Circuits 52 (2017) 2488 (DOI: 10.1109/JSSC.2017.2718665).

[27] M.F. Snoeij, et al.: "Multiple-ramp column-parallel ADC architectures for CMOS image sensors," IEEE J. Solid-State Circuits $\mathbf{4 2}$ (2007) 2968 (DOI: 10.1109/JSSC.2007.908720).

[28] S. Park, et al.: "Low-power, bio-inspired time-stamp-based 2-D optic flow sensor for artificial compound eyes of micro air vehicles," IEEE Sensors J. 19 (2019) 12059 (DOI: 10.1109/JSEN.2019.2938559).

[29] Product datasheet XT11X, https://www.klaruslight.com/ upfile/PDF/XT11X.pdf.

[30] O. Kumagai, et al:: "A 1/4-inch 3.9Mpixel low-power event-driven back-illuminated stacked CMOS image sensor," ISSCC Dig. Tech. Papers (2018) 86 (DOI: 10.1109/ISSCC.2018.8310196).

[31] K. Nie, et al.: "A single slope ADC with row-wise noise reduction technique for CMOS image sensor," IEEE Trans. Circuits Syst. I, Reg. Papers 67 (2020) 2873 (DOI: 10.1109/TCSI.2020.2979321).

[32] I. Park, et al:: "A $640 \times 640$ fully dynamic CMOS image sensor for always-on operation,” IEEE J. Solid-State Circuits 55 (2020) 898 (DOI: 10.1109/JSSC.2019.2959486).

[33] S. Kawahito: "Column readout circuit design for high-speed lownoise imaging,” IEEE ISSCC Image Sensor Forum (2010).

[34] M. Kwon and B. Murmann: "A new figure of merit equation for analog-to-digital converters in CMOS image sensors," 2018 IEEE ISCAS (2018) 1 (DOI: 10.1109/ISCAS.2018.8351578). 